\title{
On Some Probabilistic Aspects of Diffusion Models for Tissue Growth
}

\author{
Jozef Kisel'ák $^{1}$, Kamal Raj Pardasani ${ }^{2}$, Neeru Adlakha ${ }^{2}$, Milan Stehlík ${ }^{1,{ }^{*}}$ and Mamta Agrawal $^{2}$ \\ ${ }^{I}$ Department of Applied Statistics, Johannes Kepler University in Linz, Austria \\ ${ }^{2}$ Maulana Azad National Institute of Technology, Bhopal, India
}

\begin{abstract}
Understanding of tissue growth is in its nature multidisciplinary, since it varies from cancer diagnostics, image processing, fractal analysis to regular and non-regular heat flows. By medical sciences it was requested to better understood the tissue grow relation to mathematical modelling (stochastic geometry, fractal growth, diffusions). It is clear, that deterministic fractal is not an appropriate model for cancer growth. Stochastic fractal is more appropriate, however, a validation measure should be developed for better comparability with advanced stochastic geometry model, e.g. Quermass-interaction process. Moreover, relation temperature-geometry of the tissue is studied. We have partial results, where it is observed, that benign alterations and malignant tumors originating from glandular tissues (e.g.mammary, prostatic, pancreatic) are naturally modelled by non-standard diffusions. For standard diffusions, fair approximation is provided by analytical models based on convective heat transfer in infinite tissues volume (e.g. model given by Perl 1962, later extended by [1]).
\end{abstract}

Keywords: Cancer, diffusion, temperature, tissue growth.

\section{INTRODUCTION}

Tumor growth has become a paradigmatic example of multidisciplinary research, where biology and mathematics have met each other to deal with a multiscale problem. Cancer is often characterized as a chaotic, poorly regulated growth. Not surprisingly, the irregular shapes of cancerous cells, tumors, and vasculature defy description by traditional Euclidean geometry, which is based on smooth shapes such as the line, plane, cylinder, and sphere [1]. In contrast, fractal geometry reveals how an object with irregularities of many sizes may be described by examining how the number of features of one size is related to the number of similarly shaped features of other sizes. Moreover, it was observed in several studies, e.g. [2] that more natural structure is multifractality, since we expect individual variations of box counting dimensions within the sample (see e.g. [3]).

Stochastic fractal is more appropriate, however, a validation measure should be developed for better comparability with advanced stochastic geometry model, e.g. Quermassinteraction process. Anyhow, several statistical procedures can be developed on stochastic geometry arguments, see e.g. [4]. By focusing on the irregularity of tumor growth rather than on a single measure of size such as diameter or volume, fractal geometry is well suited to quantify those morphological characteristics that pathologists have long used in a qualitative sense to describe malignancies-their ragged border with the host tissue and their seemingly random patterns of

*Address correspondence to this author at the Department of Applied Statistics, Johannes Kepler University in Linz, Altenberger Straße 69, A-4040 Linz a. D., Austria; Tel: +43732 2468 6806, Fax: +43 7322468 9846; Email: Milan.Stehlik@jku.at, vascular growth. It is known that fractal geometry can be appropriate tool for describing some pathological architectures of tumors, or maybe more surprisingly, mechanism of tumor growth and angiogenesis that complement those obtained by modern molecular methods. The directional motility (chemotaxis) of cancer cells is an important aspect of metastasis.

We should consider a mathematical model which assumes that the underlying space is not smooth in some sense. For example there is a large literature on PDEs on domains with nonsmooth boundary. But most of the results require assuming boundary to be Lipschitz; this is just barely nonsmooth, since a Lipschitz boundary has a tangent plane almost everywhere. The area of fractal analysis deals with analytic question in which the underlying space has fractal structure. There exists a well developed theory of Laplacians on a class of fractals including the familiar Sierpinski gasket. As far as is known there are two main approaches. This theory may be obtained indirectly through the construction of probabilistic processes analogous to Brownian motion, and the Laplacian emerges fully formed, but not in a truly constructive manner. We refer works such as [5-8]. The disadvantage is that it does not provide a method to actually do computations. The second approach, based on calculus was introduced by Jun Kigami, see [9, 10]. In calculus, operations on functions can be defined as limit of discrete analougues. On Sierpinski gasket and related fractals, the basic operations as Laplacian, normal derivative, energy can be defined as limits of discrete operations on a sequence of graphs whose vertices approximate the fractal, see the tutorial of Strichartz [11]. It is well-known that there is a one to one correspondence between symmetric diffusion processes 
and the self adjoint operators which are generators of them. It is also well-known that they have a one to one correspondence to some quadratic forms called Dirichlet forms. To define a Laplacian $\Delta$ on a fractal $F$, one needs such a form which is a analogue of $\int|\nabla f|^{2} d x$ on $\Re^{n}$, and a measure on F.

Since then, for the last several decades, diffusion processes (and the corresponding self-adjoint operators) have been constructed on various classes of fractals and their properties have been deeply studied. It is now getting clear that the diffusions on fractals have completely different properties from diffusions on Euclidean spaces. For instance, it is understood that such processes typically have subdiffusive behaviour and heat kernels for Brownian motion on 'nice' fractals enjoy sub-Gaussian estimates.

Paper is organized as follows. First we introduce fractals and self-similarity. In particular, random self-similar sets are introduced. Stochastic diffusion over the fractal set is introduced. Then analytic approach is given in Section 3 via Dirichlet form techniques. In particular PDE for diffusion and heat flow is introduced together with recent development of heat flow modelling in human tissues. Model of tumor growth is given and anomalous diffusion introduced.

\section{SELF-SIMILAR SETS AND FRACTALS}

There occur (physical) phenomena on those objects modeled by fractals. The pioneering work in this field is by mathematical physicists who have tried to analyze properties of disordered media such as heat transfer and wave transfer. Examples of disordered media are: polymers and networks where the objects are deterministic; phenomena like growth of molds and crystals where the objects are random. How can we describe them? More precisely, how does the heat diffuse on fractals and how does a material with such structure vibrate? Since fractals do not have any smooth structures, to define differential operators is not possible from the classical viewpoint of analysis. The biggest problem is how to treat such physical phenomena in a rigorous way. To overcome such difficulty is a new challenge in mathematics.

Fractal sets and measures are mathematical models of non-integer dimensional sets and "grey-scale" images satisfying certain scaling properties. Mandelbrot introduced the term fractal and developed the connection between these ideas and a range of phenomena in the physical and biological sciences. A mathematical fractal looks the same at all scales of magnification. This is an approximation to physical fractals which appear similar to the original object only for a certain range of scales. A theory of (self-similar) fractal sets and measures and the notion of a scaling operator (iterated function system) was developed by Hutchinson in [12]. Sets with non-integral Hausdorff dimension (fractals) when they have the additional property of being in some sense either strictly or statistically self-similar, have been used extensively by Mandelbrot and others to model various physical phenomena. The key notion is "self-similar structure", which is a description of a self-similar set from a purely topological viewpoint. As we will explain immediately, topological structure of a self-similar set is essential in constructing ana- lytical structure like Laplacians and Dirichlet forms (Markovian closed symmetric forms on $L^{2}$ space studied in abstract potential theory). More precisely, if two self-similar sets are topologically same (i.e. homeomorphic), then analytical structure on one self-similar set can be transferred to another self-similar set through the homeomorphism. From the viewpoint of analysis, only the topological structure of a self-similar set is important. For example, suppose you want to know what is analysis on the Koch curve. There exists a natural homeomorphism between $[0,1]$ and the Koch curve. Through this homeomorphism, any kind of analytical structure on $[0,1]$ can be translated to its counterpart on the Koch curve. So it is easy to construct analysis on the Koch curve. The Sierpinski carpet is a fractal subset of $\Re^{2}$ defined as the fixed point of a family of eight contraction maps. We can equivalently construct the fractal by taking $[0,1]^{2}$, dividing it into nine equal squares of side length $1 / 3$, and removing the central square. This procedure is then repeated for each of the eight remaining squares and iterated infinitely. The carpet is the resulting fractal and has Hausdorff dimension $d_{f}=\ln 8 / \ln 3$. A fundamental geometrical property of this set is its infinite ramification, in that any connected subset of the fractal can only be disconnected from the rest by removing a set of dimension 1. This makes analysis on this set much more difficult than for the case of the Sierpinski gasket.

\subsection{Random Self-Similar Sets}

Sets with non-integral Hausdorff dimension are called fractals by Mandelbrot. Such sets, when they have the additional property of being in some sense either strictly or statistically self-similar, have been used extensively by Mandelbrot and others to model various physical phenomena. In paper [12] author set up a theory of (strictly) self-similar objects. We say the compact set $K \subset \Re^{n}$ is invariant with respect to $S$ if there exists a finite set $S=\left\{S_{1}, \ldots, S_{N}\right\}$ of contraction maps on $K$ such that

$$
S=\bigcup_{i=1}^{N} S_{\mathrm{i}} K
$$

It turns out, somewhat surprisingly at first, that the invariant set $K$ is determined by $S$. In fact, for given $S$ there exists a unique compact set $K$ invariant with respect to $S$. Furthermore, $K$ is the limit of various approximating sequences of sets which can be constructed from $S$. However, the fractal objects (processes) we encounter in nature are usually not generated by exact mathematical rules. The most fractal-looking natural object are not, however, precisely self-similar but are self-affine. If the similarity is not described by deterministic laws stochastic resemblance criteria can be found. Such an object is said to be randomly (statistically) self-similar.

The term random fractal is used for these fractal structures to underline their statistical character. For this let $(\Omega$, A, $\Sigma$ ) be the underlying probability space and $S=\left\{S_{1}, \ldots, S_{N}\right\}$ be a random variable. We write $\boldsymbol{S}=\operatorname{dist} S$ for the probability distribution determined by $S$. By $z$ we denote equality at the probability distribution level. If $K$ is a random set, then the random set $S K$ is defined (up to probability distribution) by 
$S K=\bigcup_{i=1}^{N} S_{\mathrm{i}} K^{(i)}$

where $S, K^{(1)}, \ldots, K^{(\mathrm{N})}$ are independent of one another and $K(i)=d K$. We say $K$ is a selfsimilar random fractal set, if $S K$ $: d K$. Beginning with a (nonrandom) set $K_{-} 0$ one defines a sequence of random sets

$S K_{0}=\bigcup_{i} S_{\mathrm{i}} K_{0}$

$S^{2} K_{0}=\bigcup_{i, j} S_{i} \circ S_{j}^{i} K_{0}$

$S^{3} K_{0}=\bigcup_{i, j, k} S_{i} \circ S_{j}^{i} \circ S_{k}^{i j} K_{0}$

etc.; where $S^{i}$ are independent of each other and of $S$, the $S^{i j}$ are independent of each other and of $S$ and the $S^{i}$, etc.

\subsection{Probabilistic Approach}

Motivated by studies in physics, Kusuoka [7] and Goldstein [8] independently construdcted "Brownian motion" on Sierpinski gasket (SG). First they considered a sequence of random walks on the graphs which approximate the SG and showed that by taking a certain scaling factor, those random walks converged to a diffusion process on $\mathrm{SG}$. Let $V_{0}=$ $\left\{p_{1}, p_{2}, p_{3}\right\}$ be a set of vertices of an equilateral triangle in complex plane. Define $f_{i}(z)=1 / 2\left(z+p_{i}\right)$ for $i=1,2,3$. The SG $\mathrm{K}$ is the unique non-empty compact subset $K$ of $\mathfrak{R}$ that satisfies

$$
K=f_{1}(K) \cup f_{2}(K) \cup f_{3}(K)
$$

Define a sequence of finite sets $\left\{V_{m}\right\}_{m \geq 0}$ inductively by $V_{m+1}=f_{1}\left(V_{m}\right) \cup f_{2}\left(V_{m}\right) \cup f_{3}\left(V_{m}\right)$. Then we have the natural graph $G_{m}$ whose set of vertices is $V_{m}$. For $p \in V_{m}$, let $V_{m, p}$ be the collection of the direct neighbors of $p$ in $V_{m}$. Let $X^{m}$ be the simple random walk on $G_{m}$ (if a particle is at $\mathrm{p}$ at time $t$, it will move to one of the direct neighbors with probability $\#\left(V_{m, p}\right)^{-1}$ at time $\left.t+1\right)$. What they proved was that

$X_{G^{-m} t}^{m} \rightarrow X_{t}$

as $m \rightarrow \infty$, where $X t$ was a diffusion process, called Brownian motion, on the SG. In this approach, a Lapacian is infinitesimal generator of the semigroup which is associated with the diffusion process. Lindstrom in [13] extended this construction of Brownian motion to nested fractals, which is a class of finitely ramified self-similar sets with strong symmetry (roughly speaking, finitely ramified self-similar sets are the self-similar sets which become disconnected if one remove a finite number of points).

The previous work on infinitely ramified fractals has concentrated on generalised Sierpinski carpets with exact self-similarity. In a series of papers $[14,15]$, the existence and properties of a Brownian motion, an isotropic diffusion process, on the two dimensional carpet were determined. This process was defined as the weak limit of a sequence of reflected Brownian motions on a sequence of subsets of $\mathfrak{R}^{2}$ converging to the fractal. Using this probabilistic approach it is possible to examine the Laplacian and the heat kernel on the fractal as these are respectively, the infinitesimal generator and transition density of the Brownian motion.

\section{ANALYTIC APPROACH}

Here we consider slightly different approach, using an idea of Dirichlet form techniques. The Dirichlet form methods have been widely applied to finitely ramified fractals and, for infinitely ramified fractals. The approach of [16] is to define a series of graph approximations to the fractal and consider the sequence of Poincaré constants generated by the Dirichlet forms on the graph approximations.

\section{A Quick View of the Theory of Dirichlet Forms}

Let $X$ be locally compact separable metric space and $v$ be a positive Radon measure on $X$ whose support is $X$. Let $\varepsilon$ be a symmetric bilinear closed form with a domain $D(\varepsilon)$ which is dense in $L^{2}(X, v)$. Pair $(\varepsilon, D(\varepsilon))$ is called a Dirichlet form if it is a Markovian (it has unit contraction property), i.e. for each $u \in D(\varepsilon), v:=\sup (0, \inf (1, u))$ and $\varepsilon(v, v) \leq \varepsilon(u, u)$. Define a new symmetric form $\varepsilon_{\alpha}(\cdot, \cdot)=\varepsilon(\cdot, \cdot)+\alpha\|\cdot\|_{L^{2}}^{2}$, for all $\alpha>0$. A Dirichlet form $(\varepsilon$, $\mathrm{D}(\varepsilon))$ is regular if there exist $\mathrm{C} \subset D(\varepsilon) \cap C_{0}(X)$ s.t. $C$ is dense in $D(\varepsilon)$ with $\varepsilon 1$-norm and $C$ is dense in $C_{0}(X)$ under the uniform norm. Pair $(\varepsilon, D(\varepsilon))$ is local if for each $u, v \quad(\varepsilon$, $D(\varepsilon)$ ) whose supports are disjoint compact sets, $\varepsilon(u, v)=0$. There is a one to one correspondence between regular Dirichlet forms on on $L^{2}(X, v)$ and $v$-symmetric Hunt processes on $X$ except for some exceptional set of starting points. Further, if the regular Dirichlet form is local, then the corresponding process is a diffusion process (i.e. Hunt process with continuous paths).

Remark 3.1. The domain of the Dirichlet form on $\mathfrak{R}^{n}$ determined by the second order differential operator is $\operatorname{Lip}(1,2, \infty)\left(\mathfrak{R}^{n}\right)$. See [17] for the fact that the domain of the Dirichlet form on SG is $\operatorname{Lip}\left(d_{w} / 2,2, \infty\right)(K)$. This fact supports that the Laplace operators on fractals are "differential operators of order $d_{w}$ ". Here $d_{w}=\ln (N+3) / \ln 2$ for the $N$ dimensional SG.

For example, if we think of the usual Brownian motion in Euclidean space $\mathfrak{R}^{n}$, the associated Dirichlet form is given by $\varepsilon(u, v)=\frac{1}{2} \int_{\Re^{n}}\langle\nabla u, \nabla v\rangle d x$.

The construction of the process associated with a given Dirichlet form, when it is possible, follows a path that looks something like this:

$(\varepsilon, D(\varepsilon)) \rightarrow\left(T_{t}\right)_{t \geq 0} \rightarrow\left(p_{t}\right)_{t \geq 0} \rightarrow\left\{X_{t}\right\}_{t \geq 0}$ 
In [18] authors considered homogeneous random Sierpinski carpets, a class of infinitely ramified random fractals which have spatial symmetry but which do not have exact self-similarity. For a fixed environment they constructed "natural" diffusion processes on the fractal and obtain upper and lower estimates of the transition density for the process that are up to constants best possible. They proved that the sequence of Poincaré constants generated by the Dirichlet forms on the graph approximations can be used to normalize the Dirichlet forms to obtain the existence of a limiting Dirichlet form which is local and regular, and hence there is an associated continuous strong Markov process.

Let $F \subset \mathfrak{R}^{D}$ be a bounded fractal set. In order to define the heat content and partition function we need to define the outer boundary of the set $F$. For each closed set $A$, let $\operatorname{Cov}(A)$ be the set of points covered by $A$. The outer boundary of the fractal is defined to be $\partial F=\partial \operatorname{Cov}(F)$. The heat content of the carpet is the total heat energy in the carpet at a given time due to unit boundary conditions and zero initial conditions within the carpet. Let $\varphi: \partial F \rightarrow[0, \infty)$ be bounded and measurable. We write $\Delta_{F}$ for the Laplace operator on $F$ and consider the following partial differential equation in $F$. Let $T: F \times[0, \infty) \rightarrow[0, \infty)$ satisfy

$$
\begin{array}{ll}
\frac{\partial T}{\partial t}=\Delta_{F} T, & x \in F \backslash \partial F, t>0, \\
T(x, 0)=0, & x \in F \backslash \partial F, \\
T(x, t)=\varphi(x), & x \in \partial F, t>0
\end{array}
$$

This equation has a probabilistic representation for its solution. Let $\left\{X_{t}\right\}$ be the stochastic process with generator $\Delta_{F}$ on the set $F$ and let $T_{A}=\inf \left\{t \geq 0: X_{t} A\right\}$. Then the solution to (3.1) can be written as

$$
T(x, t)=\mathrm{E}_{X}\left[\varphi\left(X_{T_{\partial F}}\right) I_{\left\{T_{\partial F}<t\right\}}\right] .
$$

The heat content $E_{F}(t)$ of $F$ at time $t$ is given by setting $\varphi(x)=1$ for all $x \in \partial F$ and defining

$$
E_{F}(t)=\int_{F} T(x, t) \mu(d x),
$$

where $\mu$ is the $d f$-dimensional Hausdorff measure on the fractal $F$ normalized so that $\mu(F)=1$.

\subsection{PDE for Diffusion and Heat Flow}

We restrict our attention only for two dimensional case, i.e. let $\Omega \subset \Re^{2}$ with a Lipschitz continuous boundary $\partial \Omega$. Equation

$$
B \frac{\partial u}{\partial t}=\nabla \cdot(D \nabla u)+f(t, x, u), x \in \Omega, t>0
$$

where $u$ can stand e.g. for concentration or temperature, describes diffusion (rhs) and heat flow (lhs). Additionally there are known initial and some kind of boundary conditions. Notice that generally diffusion constant $D$ can depend on unknown function $u$ and if $D$ is constant, then diffusion operator becomes simply $\Delta$.

\section{PDE for Heat Flow in Human Tissues}

In human body thermal behaviour changes under various physical and physiological conditions, which is of great interest to biomedical scientists. In order to know the thermal responses in human body organs Pennes [19] in 1948 developed the bio-heat equation to calculate the steady state temperature distribution in cylindrical shaped human arm. Later Perl [20] in 1962 has developed model of bio-heat equation by assuming all parameters as constant.

(Perl 1962) used equation (3.2) to investigate solution of some simple problems of infinite tissue medium considering the whole skin as a single layer and the parameters as constants. The model have been developed with the assumptions:

- The biological tissue is isotropic and homogeneous

- The physical properties of the tissue are independent of the tissue temperature

- $\quad$ Arterial blood temperature is constant at $37^{\circ} \mathrm{C}$

- The metabolic heat generation rate is constant per unit volume and unit time

- $\quad$ The blood perfusion rate is uniformly spatially and temporally and independent of tissue temperatur

Equation (3.2) in [1] was considered with

$u=T, B=\rho c, D=K, f=M\left(T_{A}-T\right)+S$

where $M=\rho_{b} c b \varphi_{A}$. Here, $\rho, c, K$ and $S$ are respectively the density, specific heat, thermal conductivity and rate of metabolic heat generation in tissues. Also, $\rho_{b}, c_{b}, T_{A}$ and $\varphi_{A}$ are the density of blood, specific heat of the blood, arterial blood temperature and tissue perfusion due to arterial blood. Here $\mathrm{S}$ denote the self controlled metabolic heat generation.

The boundary condition for the heat transfer occurring at skin surface is generally included in one of the following kinds of conditions, see [21]:

1. Dirichlet condition (constant temperature):

$T_{\text {skin }}=T_{\infty}$

2. Neumann condition (specified heat flux):

$-\left.K \frac{\partial T}{\partial n}\right|_{\text {skin }}=q_{s}$

3. Convective condition:

$-\left.K \frac{\partial T}{\partial n}\right|_{\text {skin }}=h\left(T_{\infty}-T\right)$

4. Radiation condition:

$-\left.K \frac{\partial T}{\partial n}\right|_{\text {skin }}=\varepsilon \sigma\left(T_{\infty}^{4}-T^{4}\right)$

where $n$ is the outward normal at the boundary of computational domain, $q S$ is constant heat flux, $\varepsilon$ is skin emissivity and $\sigma$ is Stefan-Boltzmann's constant in $\left[\mathrm{W} / \mathrm{m}^{2} \mathrm{~K}^{4}\right]$. Moreover, $h$ is the convective heat transfer coefficient for natural convection between skin tissue and air and $T_{\infty}$ is the temperature of the ambient temperature. 
Wissler [22] extended this model by taking into account the heat loss through respiration and counter current heat exchange between large arteries and large veins to obtain the temperature profile of the entire body. Cooper and Trezek [23] made an attempt to obtain analytical solution by assuming all parameters constant. Chao et al. [24, 25] investigated the steady and unsteady models with all the parameters as constant. Mitchell et al. [26] developed an analytical model to predict temperature levels as function of time in human legs. Song et al. [27] have developed a combined micro and macro vascular model for whole limb heat transfer. Some experimental attempts have been carried out by Patterson [28]. Saxena et al. [29-31] have developed one and two dimensional model of temperature distribution in human body tissues using different techniques like finite element method (FEM), analytical method and pseudo-analytic method etc. Pardasani and Adlakha [32] have developed two dimensional model in dermal regions of human limbs by using coaxial circular elements. They have also developed a model to obtain exact solution of heat flow problem in peripheral tissue layers with a solid tumor in dermis regions of human limb [33]. Agrawal et al. [1, $34,35]$ have developed two and three dimensional model to study temperature variation in dermal regions of nontapered and tapered elliptical shaped human limbs. However, all these works have been concentrated only for regular shape human organs but in reality human limb is not throughout regular tapered cylindrical in shape it may be considered irregular tapered in shape. In view of above three dimensional steady state model has been developed to study temperature distribution in dermal regions of irregular tapered cylindrical shaped human limbs. The structure of the limb is not same throughout, considering the shape of the upper limb may be tapered, nontapered, elliptical or circular [36]. For those regions where it resembles with elliptical shape have eccentricity greater than zero and less than one and where it resembles with circular shape has the eccentricity zero. The radius and eccentricity varies according to the shape of the limb. In order to incorporate circular and elliptical shapes at different axial positions, the eccentricity and radius of the limb is taken variable along axial direction. Hence radius can be taken as a $l$-th degree polynomial of $\boldsymbol{Z}$ as shown below where $/$ can be any finite positive integer. The different values of $/$ will give different shapes of the limb [1].

After developing the mathematical model for temperature distribution in SST region of irregular tapered shaped human limbs, incorporated the values of the different parameters involved through various experimental papers $[11,26]$. Finite element technique has been used as in our previous paper [1] to solve the mathematical model, computed the results by using Matlab 7.5 and obtain the graphs to validate the results with the physiology of the upper limb.

Fig. (1a) to (e) are the two dimensional graphs between angular coordinates and temperatures. Fig. (1f) is three dimensional graphs between angular coordinates, radial coordinates and temperatures. In these graphs the temperature falls along radial direction and effect of boundary condition on temperature distribution is also observed along radial direction. This effect of boundary condition on temperature distribution is observed to be decreasing along radial direction. This may be because the most of the heat is transported from core of the limb through SST regions along radial di- rection to the outer surface of the limb from where it is lost to the environment. Also the change in the slope is observed at junctions of tissue layers along radial direction. This may be due to different physical and physiological properties of each layer. Also we see that as soon as we move from one end to another end of the limb along axial direction temperature decreases. This is due to change in the shapes of the human limb at different axial position in a irregular tapered shape human limb. The change in the shape causes the change in the area of the limb expose to the environment thereby causing the change in heat transport in the dermal tissues and heat loss from the outer surface to the environment. The results obtained indicate that the effect of shape and size of different portions of limbs have significant effect on temperature distribution in dermal layers (SST) of human limbs. The finite element as proved to be quite powerful and effective in modelling the problem of temperature distributions in dermal layers of irregular tapered shaped human limbs as it was possible to deal with nonregular structures in terms of combination of multiple regular substructures.

\section{Model of Tumor Growth}

For simplicity we consider a model, see [37], depending on

- the nutrient concentration $\sigma$

- the internal pressure $p$ of the proliferating tissue

but does not on

- the concentration of inhibitors

- the effect of angiogenesis

- the presence of the necrotic zone

and the proliferation rate is linear i.e. $\mu(\sigma-\tilde{\sigma}), \mu>0$. The tumor receives nutrients throught the boundary, where the concentration is a constant $\hat{\sigma}>\tilde{\sigma}$. The diffusion equation (3.2) contains

$u=\sigma, B=c, D=1, f=-\lambda \sigma$,

where $\Omega=\Omega_{t}$ is evolving tumor region and $c>0, \lambda>0$. By the conservation of mass we have

$\Delta p-\mu(\sigma-\tilde{\sigma})=0$ in $\Omega_{t}$,

and the boundary conditions on $\partial \Omega_{t}$ are

$$
\begin{aligned}
& \sigma=\hat{\sigma}, \\
& p=\gamma \kappa, \\
& \frac{\partial p}{\partial n}=-V_{n} .
\end{aligned}
$$

Two boundary conditions overdetermine the problem and allow one to find the free boundary position and velocity at time $t$. The initial conditions will be determined by measurements from real "data".

Sometimes one need to speed-up (superdiffusion) or slow-down (subdiffusion) the diffusion, it is possible with the help of so-called $p$-Laplacian $\cdot(|u| p-2 u), 1<p<\infty$ an value $p<2, p>2$ respectively or use some kind of nonlin- 

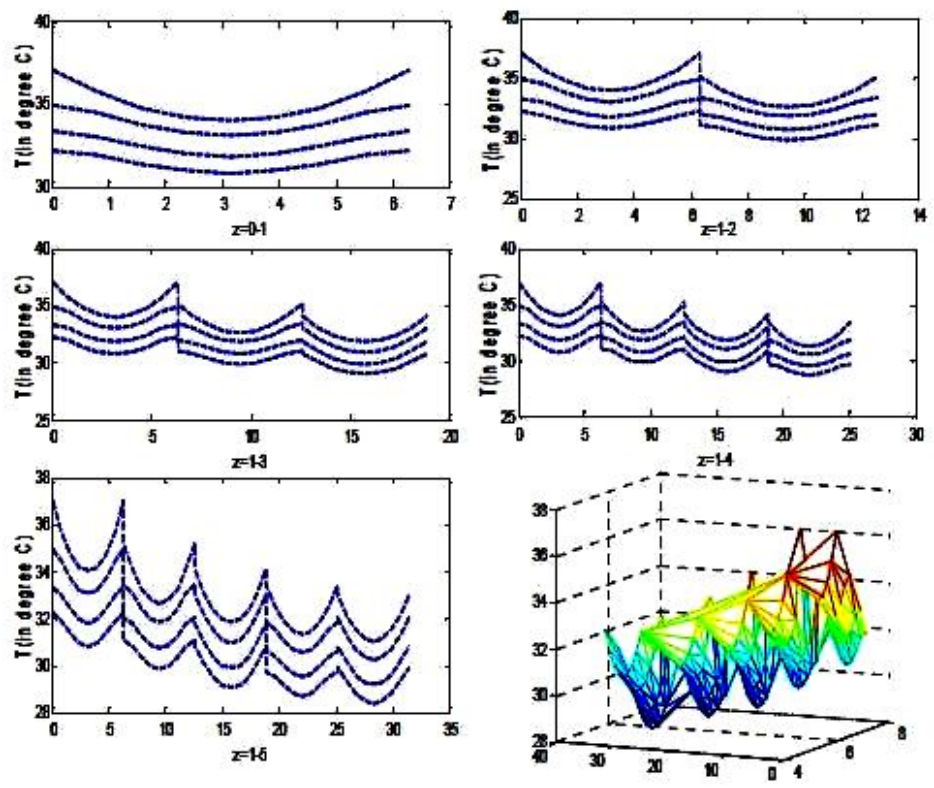

Fig. (1). Fig. 1(a)-1(f).

ear diffusion. Authors in [38] generalized the classical KS model in this fashion.

\section{Generalized Keller-Segel Model with Power Law Diffu- sion: Tsallis Entropy}

Authors in $[39$, p. 256] consider a generalized class of models describing the chemotaxis of biological populations. In the simplest model, the diffusion coefficient and $D$ and the mobility $\chi$ of the bacteria are constant. However, the original Keller-Segel model allows these coefficients to depend on concentration of the bacteria and of the chemical. E.g. they mention equation (3.2) with $u=\rho, f=\nabla \cdot(\chi \rho \nabla c)$, but with nonlinear operator $D \nabla \rho^{q}$ instead of $D \nabla \rho$. It corresponds to a power law diffusion $D(\rho)=D \rho^{q-1}$ and one can assume a constant mobility $\chi(x)=\chi$. This leads to the stochastic process, ordinary Ito-Langevin equation

$\frac{d r}{d t}=\chi \nabla c+\sqrt{2 D} \rho^{\frac{q-1}{2}} R(t)$

where $R(t)$ is a white noise. Notice that constant diffusion $D(\rho)=D$ leads to standard Keller-Segel model related to Boltzmann entropy. This model takes into consideration effects of non-ergodicity and nonextensitivity. It eventuates in a situation of anomalous diffusion (non-linear relationship to time). For $q \neq 1$, the size of the random kicks is not uniform and a praticle which is in region with large $\rho(r, t)$ will tend to have larger (smaller) kicks if $q>1(q<1)$. This leads to the fractal phase structure. The Tsallis entropy

$S(t)=-\frac{1}{q-1} \int\left(\rho^{q}-\rho\right) d r$ associated to equation (3.2) with a power law diffusion is the polynomial functional. In the case $q=2$, the quadratic functional gives the stationary solution, which possesses linear relationship between the density and the concentration.

\subsubsection{Numerical analysis of heat equation with p- Laplacian}

Assume $p \in(1, \infty), \Omega \subset \Re^{d}, d \geq 1$ with a Lipschitz continuous boundary $\partial \Omega$ in the case $d>1$.

$$
\begin{aligned}
& \frac{\partial u}{\partial t}=\nabla \cdot\left(|\nabla u|^{p-2} \nabla u\right)+f, \quad x \in \Omega, t>0, \\
& u(x, 0)=0, \quad x \in \Omega, \\
& u(x, t)=u_{0}(x), \quad x \in \Omega
\end{aligned}
$$

where $u_{0} \in V=W_{0}^{1, p}(\Omega)$ and $f \in V^{*}=W_{0}^{-1, p^{*}}(\Omega)$. Define $A: V \rightarrow V^{*}$ as follows: for every $\mathrm{v} \in \mathrm{V}$,

$(A u, v)=\int_{\Omega}|\nabla u|^{p-2} \nabla u \cdot \nabla v d x$.

We say that $u$ is a weak solution of (3.7) on $[0, T]$ if $u \in$ $L^{p}(0, T ; V)$ solves the following weak problem :

$\frac{d}{d t}\langle u, v\rangle+(A u, v)=\langle f, v\rangle$,
$\langle u(0), v\rangle=\left\langle u_{0}, v\right\rangle$,

for all $v \in V$, in the sense of distribution on [0,T]. Suppose the following time discretizations. Let $\left\{t_{\boldsymbol{i}}\right\}^{\infty}$ be a uniform partition of $\mathfrak{R}^{+}$with $t_{i}=\mathrm{i} \Delta t$ for time step $\Delta t>0$. Author in [40] used the backward Euler scheme (recursive nonlinear elliptic problem): 
$v \in V$,

Given $\mathrm{f} \in V *, u_{0} \in L^{2}(\Omega)$, find $u_{i} \in V$, such that for all

$\left\langle\frac{u_{i}-u_{i-1}}{\Delta t}, v\right\rangle+\left(A u_{i}, v\right)=\langle f, v\rangle$,

$u_{0}=u_{0}(x)$,

where $u i=u i(x), i=1,2, \ldots$. Further, let $\{t n, i\} \infty$ be a uniform partition of $\mathfrak{R}^{+}$and $t_{n, i}=i \Delta t n$, with time step $\Delta t n>0$. Let $\left\{u_{n, i}\right\} \infty$ be the solution set defined by (3.10) with $\Delta t$ replaced by $\Delta t n$. Define

$u_{n}(t)=t-t_{n, I} \Delta t_{n} u_{n, i+1}+t_{n, i+1}-t \Delta t_{n} u_{n, i}, t_{n, i}<t$ $<t_{n, i+1}$

He gave a uniform convergence results in spaces $L^{2}(\Omega)$ and $V$ for $u_{n}(t) \rightarrow u$ in $C\left(\Re^{+} ; \cdot\right)$ for the backward Euler scheme. They summarized some of the convergence and stability results also for the Crank-Nicholson scheme:

$\left\langle\frac{u_{i}-u_{i-1}}{\Delta t}, v\right\rangle+\left(A \frac{u_{i}+u_{i-1}}{2}, v\right)=\langle f, v\rangle, u_{0}=u_{0}(x)$,

where $u_{i}=u_{i}(x), i=1,2, \ldots$

\section{A Semi-Discrete Finite Element Method}

For simplicity, we assume that $\Omega$ is convex with piecewise smooth boundary $\partial \Omega$ where $d \geq 1$. Let $S_{h}(\Omega)$ be a regular conforming finite element space of $V$, where $h$ is the maximum of the diameters of the elements of triangulation. Consider the following semidiscrete FEM scheme: Find $u_{h}$ $S_{h}(\Omega)$, such that for all $v_{h} \in S_{h}(\Omega)$,

$\left\langle\frac{d u_{h}}{d t}, v_{h}\right\rangle+\left(A u_{h}, v_{h}\right)=\left\langle f, v_{h}\right\rangle,\left(A u_{h}(0), v_{h}\right)=\left(A u(0), v_{h}\right)$.

Denote $\left\{U_{h, n}\right\} \infty$ as the backward Euler solution sequence for (3.13). He stated and proved their main result about uni-

form convergence of $\mathrm{U}_{h} \in C\left(\mathfrak{R}^{+} ; \mathrm{S}_{h}(\Omega)\right)$ to $u \in C\left(\mathfrak{R}^{+} ; V\right)$ for $t \mathfrak{R}^{+} \cup\{\infty\}$ as $h \rightarrow 0$.

\section{CONCLUSIONS}

As we realized in the [41], the landmarks (points of extremal process) are hardly independent. The one possible dependence structure is pseudoexponential introduced in [42]. Therein we also proposed and motivated the fully parametric model of landmark dependency. Moreover, we derive statistical methods for inference on its parameters. In particular we develop in this paper exact likelihood ratio test on scale (Hausdorff dimension in the case of fractal cancer model). We also develop a GOF test for pseudoexponential dependence versus independence. For future research it will be practical to provide an analytical tool for discrimination between cancer and healthy ranges of fractal dimensions of tissues. Beside that implementation of principles of temperature distribution modeling in software, e.g. Ansys, could be of practical use (see e.g.[43]).

The authors aim only to present some interesting ideas how to model tumor growth pattern. As pointed by referee, the model overly simplifies the tumor dynamics and it is not clear which setting it can be applied to. Tumor growth rate can be affected by multiple microenvironment factors such as the growth factors. The tumor shape (boundaries) quantification also depends on how the shape is defined (by what type of image modality) and which smoothing technique is used. For example, the tumor shape from PET image is less clearly defined than the shape from CT imaging. More work will be needed to better understand dynamics of tumor growth.

\section{ACKNOWLEDGEMENTS}

Authors acknowledge support of WTZ Project Nr. IN 11/2011. We thank the Editor and Reviewers whose insightful comments helped us to sharpen the paper.

\section{REFERENCES}

[1] M. Agrawal, N. Adlakha and K. R. Pardasani, "Three dimensional finite element model to study heat flow in dermal regions of elliptical and tapered shape human limbs", Appl. Math. Comput., vol. 217, pp. 4129-4140, December 2010.

M. Stehlik, F. Wartner and M. Minarova, "Fractal analysis for cancer research: case study and simulation of fractals", Pliska, vol. 22, pp. 195-206, 2013

L. E. George and K. H. Sager, "Breast Cancer Diagnosis Using Multi-Fractal Dimension Spectra", in IEEE International Conference on Signal Processing and Communications, 2007, p. 592-595.

[4] M. Stehlik, T. Mrkvicka, J. Filus and L. Filus, "Recent development on testing in cancer risk: a fractal and stochastic geometry", $J$ Reliab Stat Stud, vol. 5, pp. 83-95, 2012.

[5] M. T. Barlow and E. A. Perkins. "Brownian motion on the Sierpinski gasket”, Probab. Theor. Relat. Field., vol. 79, no. 4, pp. 543623,1988 .

[6] S. Goldstein, "Random walks and diffusions on fractals", in Percolation Theory and Ergodic Theory of Infinite Particle Systems, vol. 8, IMA Math Appl., H. Kesten, Ed. New York: SpringerVerlag, 1987, pp. 121-129.

[7] S. Kusuoka, "A diffusion process on a fractal", in Probabilistic Methods in Mathematical Physics, Boston: Academic Press, 1987, pp. 251-274.

[8] S. Kusuoka, "Dirichlet forms on fractals and products of random matrices", Publ. Res. Inst. Math. Sci., vol. 25, pp. 659-680, 1989.

[9] J. Kigami, "A harmonic calculus on the Sierpinski spaces", Jpn J. Appl. Math., vol. 6, pp. 259-290, 1989.

[10] J. Kigami, "Harmonic calculus on p.c.f. self-similar sets", Trans. Am. Math. Soc., vol. 335, pp. 721-755, 1993.

[11] R. S. Strichartz, Differential Equations on fractals: A Tutorial, Princeton, NJ : Princeton Univ. Press, 2006.

[12] J. E. Hutchinson, "Fractals and self similarity", Ind. Univ. Math. J., vol. 30 , pp. 713-747, 1981.

[13] T. Lindstrom , "Brownian motion on nested fractals". in Mem. Amer. Math. Soc., vol. 420, Providence, RI, 1990.

[14] M. T. Barlow and R. F. Bass, "Construction of Brownian motion on the Sierpinski carpet”, Ann. Inst. H. Poincare, vol. 25, pp. 225 257, 1989.

[15] M. T. Barlow and R. F. Bass, "On the resistance of the Sierpinski carpet”, Proc. R. Soc. London A., vol. 431, pp. 345-360, 1990.

[16] S. Kusuoka and X. Y. Zhou, "Dirichlet forms on fractals: Poincaré constant and resistance", Prob. Theor. Rel. Field., vol. 93, pp. 169$196,1992$.

[17] A. Jonsson, "Brownian motion on fractals and function spaces", Math. Z., vol. 222, pp. 496-504, 1996.

[18] B. M. Hambly, T. Kumagai, S. Kusuoka and X. Y. Zhou, "Transition density estimates for diffusion processes on homogeneous random Sierpinski carpets", J. Math. Soc. Jpn, vol. 52, pp. 373-408, 2000.

[19] H. Pennes, "Analysis of tissue and arterial blood temperature in the resting human forearm", J. Appl. Physiol., vol. 1, no. 2, pp. 93-122, 1948 . 
[20] W. Perl, "Heat and matter distribution in body tissues and determination of tissue blood flow by local clearance methods", $J$. Theo. Biol., vol. 2, pp. 201-235, 1962.

[21] F. Filipoiu, A. I. Bogdan and I. M. Carstea, "Computer-aided analysis of the heat transfer in skin tissue", in Proceedings of the 3rd WSEAS Int. Conference on finite differences - finite elements - finite volumes - boundary elements, 2010, pp. 53-59.

[22] E. H. Wissler, "Pennes' 1948 paper revisited", J. Appl. Physiol, vol. 85 , no. 1, pp. 35-41, 1998.

[23] T. E. Cooper, and G. J. Trezek, "A probe technique for determining the thermal conductivity of tissue", J. Heat Trans., ASME, vol. 94, pp. 133-140, 1972.

[24] K. N. Chao, J. G. Eisley, and W. J., Yang, "Heat and water migration in regional skins and subcutaneous tissues", in Bio.Mech. Symp, ASME, 1973, pp. 69-72.

[25] K. N. Chao and W. J. Yang, "Response of skin and tissue temperature in sauna and steam baths", in Bio. Mech. Symp, ASME, 1975, pp. 69-71.

[26] J. W. Mitchell, T. L. Galvez, J. Hengle, G. E. Myers and K. L. Siebercker, "Thermal response of human legs during cooling", $J$. Appl. Physiol. vol .29, no. 6, pp. 859-865, 1970.

[27] W. J. Song, S. Weinbaum, L. M. Jiji, and D. Lemons, "A combined macro and micro vascular model for whole limb heat transfer”, Trans. ASME J. Biomech. Eng., vol. 110, pp. 259-268, 1988.

[28] A. M. Patterson, "Measurement of temperature profiles in human skin", S. Afr. J. Sc., vol. 72, pp. 78-79, 1976.

[29] V. P. Saxena, "Temperature distribution in human skin and subdermal tissues", J. Theo. Biol., vol. 102, no. 2, pp. 277-286, 1983.

[30] V. P. Saxena, and D. Arya, "Steady state heat distribution in epidermis, dermis and subdermal tissues", J. Theor. Biol., vol. 89, pp. 423- 432, 1981.

[31] V. P. Saxena and J. S. Bindra, "Steady state temperature distribution in dermal blood flow, perspiration and self controlled metabolic heat generation", Ind. J.Pure Appl. Math., vol. 15, no. 1, pp. 31-42, 1984

[32] K. R. Pardasani, and N. Adlakha, "Coaxial circular sector elements to study two-dimensional heat distribution problem in dermal re- gions of human limbs", Math. Comput. Modell, vol. 22, no.9, pp.127-140, 1995.

[33] K. R. Pardasani and N. Adlakha, "Exact solution to a heat flow problem in peripheral tissue layers with a solid tumor in dermis", Ind. J. Pure. Appl. Math. vol. 22, no. 8, pp. 679-682, 1991.

[34] M. Agrawal, N. Adlakha and K. R. Pardasani, "Seminumerical model to study temperature distribution in peripheral layers of elliptical and tapered shaped human limbs", J. Mech. Med. Biol., vol. 10 , no. 1 , pp. 57-72, 2010.

[35] M. Agrawal, N. Adlakha and K. R. Pardasani, "Finite element model to study thermal effect of uniformly perfused tumor in dermal layers of elliptical shaped human limb", Int. J. Biomath., vol. 4, no. 2, pp. 241-254, 2011.

[36] E. D. Yildirim and B. Ozerdem, "A numerical simulation study for the human passive thermal system", Energy Build, vol. 40, pp. $1117-1123,2008$.

[37] A. Friedman, "Free boundary problems in science and technology", Notices Am. Math. Soc., vol.47, no. 8, pp. 854-861, 2000.

[38] M. Bendahmane, R. Burger, R. Ruiz-Baier, J. M. Urbano, "On a doubly nonlinear diffusion model of chemotaxis with prevention of overcrowding", Math. Methods Appl. Sci., vol. 32, no. 13, pp. 1704-1737, 2009.

[39] P. H. Chavanis, "Generalized Keller-Segel models of chemotaxis Analogy with nonlinear mean field Fokker-Planck equations", in Chaos, complexity and transport; theory and applications; proceedings of the CCT '07, 2008

[40] N. Ju, "Numerical analysis of parabolic p-Laplacian: approximation of trajectories", Siam J. Numer. Anal., vol. 37, no. 6, pp. 1861$1884,2000$.

[41] M. Stehlik, J. Prostakova, S. Giebel and J. P. Schenk, "Statistical inference on fractals for cancer risk assessment", submitted, 2011.

[42] J. Filus, L. Filus and M. Stehlik, "Pseudoexponential modelling of cancer diagnostic testing", in Biometrie Und Medizinische Informatik Greifswalder Seminarberichte Heft 15, 2009, pp. 41-54.

[43] J. Babiak, M. Minarova and D. Petras, "Principles and calculations of temperature distribution in an active slab depending up various operation modes 20 of TABS using FEM software", in Proceeding in CLIMA Congress, 2005.

Received: August 28, 2013

Revised: September 28, 2013

Accepted: October 15, 2013

(C) Kisel'ák et al.; Licensee Bentham Open.

This is an open access article licensed under the terms of the Creative Commons Attribution Non-Commercial License (http://creativecommons.org/licenses/by-nc/3.0/) which permits unrestricted, non-commercial use, distribution and reproduction in any medium, provided the work is properly cited. 